\title{
NOTE ON QUANTUM DYNAMICAL ENTROPIES
}

\author{
LUIGI ACCARDI \\ Centro Matematico Vito Volterra, Università di Roma II, via di Tor Vergata, Roma, Italy \\ Masanori Ohya and Noboru Watanabe \\ Department of Information Sciences, Science University of Tokyo, Noda, Chiba 278, Japan \\ (Received September 12, 1996)
}

\begin{abstract}
Classical dynamical entropy is an important tool to analyse the efficiency of information transmission in communication processes. Quantum dynamical entropy was first studied by Connes, Størmer and Emch. Since then, there have been many attempts to formulate or compute the dynamical entropy for some models. Here we review four formulations due to (a) Connes, Narnhofer and Thirring, (b) Ohya, (c) Accardi, Ohya and Watanabe, (d) Alicki and Fannes. We consider mutual relations between these formulations and we show some concrete computations for a model.
\end{abstract}

\section{Introduction}

The classical dynamical (or Kolmogorov-Sinai) entropy $S(T)[13,26]$ for a measure preserving transformation $T$ was defined on a message space through finite partitions of the measurable space. The classical coding theorems of Shannon are important tools to analyse communication processes which have been formulated by the mean dynamical entropy and the mean dynamical mutual entropy. The mean dynamical entropy represents the amount of information per one letter of a signal sequence sent from an input source, and the mean dynamical mutual entropy does the amount of information per one letter of the signal received in an output system.

The quantum dynamical entropy (QDE) has been studied by Connes and Størmer [10], Emch [11], Connes, Narnhofer and Thirring [9], Alicki and Fannes [5], and others $[7,22]$. Their dynamical entropies were defined in the observable spaces.

Recently, the quantum dynamical entropy and the quantum dynamical mutual entropy were studied by one of the present authors $[14,23]$. They are formulated in the state spaces through the complexity of Information Dynamics [21, 23]. Furthermore, another formulation of the dynamical entropy through the quantum Markov chain (QMC) was done in [4].

In Section 1 we brietly review the formulation by Connes, Narnhofer and 'I'hirring (CNT). In Section 2 we explain the formulation by complexity [23, 24]. In Section 3 we 
review the formulation by QMC, and in Section 4 we briefly explain the formulation by Alicki and Fannes (AF). Mutual relations among these four formulations are discussed in Section 4, and in Section 6 we compute the mean dynamical entropies in quantum communication processes.

\section{The CNT formulation}

Let $\mathcal{A}$ be a unital $C^{*}$-algebra, $\theta$ be an automorphism of $\mathcal{A}$, and $\varphi$ be a stationary state over $\mathcal{A}$ with respect to $\theta ; \varphi \circ \theta=\varphi$. Let $\mathcal{B}$ be a finite dimensional $C^{*}$-subalgebra of $\mathcal{A}$.

The CNT entropy [9] for a subalgebra $\mathcal{B}$ is given by

$$
H_{\varphi}(\mathcal{B})=\sup \left\{\sum_{k} \lambda_{k} S\left(\omega_{k}|\mathcal{B}, \varphi| \mathcal{B}\right) ; \quad \varphi=\sum_{k} \lambda_{k} \omega_{k} \quad \text { finite decomposition of } \varphi\right\},
$$

where $\varphi \mid \mathcal{B}$ is the restriction of the state $\varphi$ to $\mathcal{B}$ and $S(\cdot, \cdot)$ is the relative entropy for $C^{*}$-algebra $[6,27,28]$.

The CNT dynamical entropy with respect to $\theta$ and $\mathcal{B}$ is given by

$$
\tilde{H}_{\varphi}(\theta, \mathcal{B})=\limsup _{N \rightarrow \infty} \frac{1}{N} H_{\varphi}\left(\mathcal{B} \vee \theta \mathcal{B} \vee \cdots \vee \theta^{N-1} \mathcal{B}\right),
$$

and the dynamical entropy for $\theta$ is defined by

$$
\tilde{H}_{\varphi}(\theta)=\sup _{\mathcal{B}} \tilde{H}_{\varphi}(\theta, \mathcal{B}) .
$$

\section{Formulation by complexity}

In this section, we first review the concepts of channel and complexity, which are the key concepts of ID (Information Dynamics) introduced by Ohya [21, 23].

Let $(\mathcal{A}, \Sigma(\mathcal{A}), \alpha(G)),(\overline{\mathcal{A}}, \bar{\Sigma}(\overline{\mathcal{A}}), \bar{\alpha}(\bar{G}))$ be an input (initial) and an output (final) $C^{*}$ systems, respectively, where $\mathcal{A}$ (resp. $\overline{\mathcal{A}}$ ) is a unital $C^{*}$-algebra, $\Sigma(\mathcal{A})$ (resp. $\bar{\Sigma}(\overline{\mathcal{A}})$ ) is the set of all states on $\mathcal{A}$ (resp. $\overline{\mathcal{A}}$ ) and $\alpha(G)$ (resp. $\bar{\alpha}(\bar{G})$ ) is the group of automorphisms of $\mathcal{A}$ (resp. $\overline{\mathcal{A}}$ ) indexed by a group $G$ (resp. $\bar{G}$ ).

The channel is a map $\Lambda^{*}$ from $\Sigma(\mathcal{A})$ to $\bar{\Sigma}(\overline{\mathcal{A}})$. If the dual map $\Lambda$ from $\overline{\mathcal{A}}$ to $\mathcal{A}$ of $\Lambda^{*}$ satisfies the complete positivity, the channel $\Lambda^{*}$ is called a complete positive channel (CP channel).

For a weak ${ }^{*}$ compact convex subset $\mathcal{S}$ of $\Sigma$, there exists a maximum measure $\mu$ with the barycenter $\varphi$ such that

$$
\varphi=\int_{\mathcal{S}} \omega d \mu
$$

The compound state, introduced in $[17,18]$, which exhibits the correlation between the initial $\varphi$ and final $\Lambda^{*} \varphi$ states is given by

$$
\mathcal{E}_{\mu}^{*} \varphi=\int_{\mathcal{S}} \omega \otimes A^{*} \omega d \mu
$$


In the sequel, we use a CP channel $\Lambda^{*}$ and the compound state to formulate the dynamical entropy.

There are two types of complexities in ID. One of them is the complexity $C_{T}^{\mathcal{S}}(\varphi)$ of the system itself, the other is the transmitted complexity $T^{\mathcal{S}}\left(\varphi ; A^{*}\right)$ from an initial system to a final system. These complexities should satisfy the following conditions:

(i) For any $\varphi \in \mathcal{S} \subset \Sigma$,

$$
C^{\mathcal{S}}(\varphi) \geq 0, \quad T^{\mathcal{S}}\left(\varphi ; \Lambda^{*}\right) \geq 0 .
$$

(ii) If there exists an orthogonal bijection $j: \operatorname{ex} \Sigma \rightarrow \operatorname{ex} \Sigma$; the set of all extremal points in $\Sigma$, then

$$
\begin{aligned}
C^{j(\mathcal{S})}(j(\varphi)) & =C^{\mathcal{S}}(\varphi), \\
T^{j(\mathcal{S})}\left(j(\varphi) ; \Lambda^{*}\right) & =T^{\mathcal{S}}\left(\varphi ; \Lambda^{*}\right) .
\end{aligned}
$$

(iii) For a state $\Psi=\varphi \otimes \psi \in \mathcal{S}_{t}$, with $\varphi \in \mathcal{S}, \psi \in \overline{\mathcal{S}}$,

$$
C^{\mathcal{S}_{t}}(\Psi)=C^{\mathcal{S}}(\varphi)+C^{\overline{\mathcal{S}}}(\psi)
$$

(iv) $0 \leq T^{\mathcal{S}}\left(\varphi ; \Lambda^{*}\right) \leq C^{\mathcal{S}}(\varphi)$.

(v) $T^{\mathcal{S}}(\varphi ;$ id $)=C^{\mathcal{S}}(\varphi)$.

Next we review the definitions of the three types of entropic complexities introduced in $[23]$.

Let $(\mathcal{A}, \Sigma(\mathcal{A}), \alpha(G)),(\overline{\mathcal{A}}, \bar{\Sigma}(\overline{\mathcal{A}}), \bar{\alpha}(\bar{G}))$ and $\mathcal{S}$ be as above. Let $M_{\varphi}(\mathcal{S})$ be the set of all maximal measures $\mu$ on $\mathcal{S}$ with the fixed barycenter $\varphi$, and let $F_{\varphi}(\mathcal{S})$ be the set of all measures having finite support with the fixed barycenter $\varphi$. Then the three pairs of complexities are

$$
\begin{aligned}
T^{\mathcal{S}}\left(\varphi ; \Lambda^{*}\right) & \equiv \sup \left\{\int_{\mathcal{S}} S\left(\Lambda^{*} \omega, \Lambda^{*} \varphi\right) d \mu ; \mu \in M_{\varphi}(\mathcal{S})\right\} \\
C_{T}^{\mathcal{S}}(\varphi) & \equiv T^{\mathcal{S}}(\varphi ; \mathrm{id}) \\
I^{\mathcal{S}}\left(\varphi ; \Lambda^{*}\right) & \equiv \sup \left\{S\left(\int_{\mathcal{S}} \omega \otimes \Lambda^{*} \omega d \mu, \varphi \otimes \Lambda^{*} \varphi\right) ; \mu \in M_{\varphi}(\mathcal{S})\right\} \\
C_{I}^{\mathcal{S}}(\varphi) & \equiv I^{\mathcal{S}}(\varphi ; \text { id }) \\
J^{\mathcal{S}}\left(\varphi ; \Lambda^{*}\right) & \equiv \sup \left\{\int_{\mathcal{S}} S\left(\Lambda^{*} \omega, \Lambda^{*} \varphi\right) d \mu_{f} ; \quad \mu_{f} \in F_{\varphi}(\mathcal{S})\right\} \\
C_{J}^{\mathcal{S}}(\varphi) & \equiv J^{\mathcal{S}}(\varphi ; \mathrm{id}) .
\end{aligned}
$$

Based on the above complexities, we explain the quantum dynamical complexity (QDC) [17].

Let $\theta$ (resp. $\bar{\theta}$ ) be a stationary automorphism of $\mathcal{A}($ resp. $\overline{\mathcal{A}}) ; \varphi \circ \theta=\varphi$, and $\Lambda$ be a covariant $\mathrm{CP}$ map (i.e., $\Lambda \circ \theta=\bar{\theta} \circ \Lambda$ ) from $\overline{\mathcal{A}}$ to $\mathcal{A}$. $\mathcal{B}_{k}$ (resp. $\overline{\mathcal{B}}_{k}$ ) is a finite subalgebra 
of $\mathcal{A}\left(\right.$ resp. $\overline{\mathcal{A}}$ ). Moreover, let $\alpha_{k}$ (resp. $\bar{\alpha}_{k}$ ) be a CP unital map from $\mathcal{B}_{k}$ (resp. $\overline{\mathcal{B}}_{k}$ ) to $\mathcal{A}\left(\right.$ resp. $\overline{\mathcal{A}}$ ) and $\alpha^{M}$ and $\bar{\alpha}_{\Lambda}^{N}$ are given by

$$
\begin{aligned}
\alpha^{M} & =\left(\alpha_{1}, \alpha_{2}, \ldots, \alpha_{M}\right), \\
\bar{\alpha}_{\Lambda}^{N} & =\left(\Lambda \circ \bar{\alpha}_{1}, \Lambda \circ \bar{\alpha}_{2}, \ldots, \Lambda \circ \bar{\alpha}_{N}\right) .
\end{aligned}
$$

Two compound states for $\alpha^{M}$ and $\bar{\alpha}_{A}^{N}$, with respect to $\mu \in M_{\varphi}(\mathcal{S})$, are defined as

$$
\begin{aligned}
\Phi_{\mu}^{\mathcal{S}}\left(\alpha^{M}\right) & =\int_{\mathcal{S}} \bigotimes_{m=1}^{M} \alpha_{m}^{*} \omega d \mu, \\
\Phi_{\mu}^{\mathcal{S}}\left(\alpha^{M} \cup \bar{\alpha}_{\Lambda}^{N}\right) & =\int_{\mathcal{S}} \bigotimes_{m=1}^{M} \alpha_{m}^{*} \omega \stackrel{\otimes}{N=1}_{n}^{N} \bar{\alpha}_{n}^{*} \Lambda^{*} \omega d \mu .
\end{aligned}
$$

Using the above compound states, the three transmitted complexities [23] are defined by $T_{\varphi}^{\mathcal{S}}\left(\alpha^{M}, \bar{\alpha}_{\Lambda}^{N}\right)=\sup \left\{\int_{\mathcal{S}} S\left(\bigotimes_{m=1}^{M} \alpha_{m}^{*} \omega \bigotimes_{n=1}^{N} \bar{\alpha}_{n}^{*} \Lambda^{*} \omega, \Phi_{\mu}^{\mathcal{S}}\left(\alpha^{M}\right) \otimes \Phi_{\mu}^{S}\left(\bar{\alpha}_{\Lambda}^{N}\right)\right) d \mu ; \mu \subset M_{\varphi}(S)\right\}$, $I_{\varphi}^{\mathcal{S}}\left(\alpha^{M}, \bar{\alpha}_{\Lambda}^{N}\right) \equiv \sup \left\{S\left(\Phi_{\mu}^{\mathcal{S}}\left(\alpha^{M} \cup \bar{\alpha}_{\Lambda}^{N}\right), \Phi_{\mu}^{\mathcal{S}}\left(\alpha^{M}\right) \otimes \Phi_{\mu}^{\mathcal{S}}\left(\bar{\alpha}_{\Lambda}^{N}\right)\right) ; \quad \mu \in M_{\varphi}(\mathcal{S})\right\}$, $J_{\varphi}^{\mathcal{S}}\left(\alpha^{M}, \bar{\alpha}_{A}^{N}\right) \equiv \sup \left\{\int_{\mathcal{S}} S\left(\bigotimes_{m=1}^{M} \alpha_{m}^{*} \omega \bigotimes_{n=1}^{N} \bar{\alpha}_{n}^{*} \Lambda^{*} \omega, \Phi_{\mu}^{\mathcal{S}}\left(\alpha^{M}\right) \otimes \Phi_{\mu}^{\mathcal{S}}\left(\bar{\alpha}_{A}^{N}\right)\right) d \mu_{f} ; \mu_{f} \in F_{\varphi}(\mathcal{S})\right\}$.

When $\mathcal{B}_{k}=\overline{\mathcal{B}}_{k}=\mathcal{B}, \mathcal{A}=\overline{\mathcal{A}}, \theta=\bar{\theta}, \alpha_{k}=\theta^{k-1} \circ \alpha=\bar{\alpha}_{k}$, where $\alpha$ is a unital CP map from $\mathcal{A}_{0}$ to $\mathcal{A}$, the mean transmitted complexities are

$$
\begin{aligned}
\tilde{T}_{\varphi}^{\mathcal{S}}\left(\theta, \alpha, \Lambda^{*}\right) & \equiv \limsup _{N \rightarrow \infty} \frac{1}{N} T_{\varphi}^{\mathcal{S}}\left(\alpha^{N}, \bar{\alpha}_{\Lambda}^{N}\right), \\
\tilde{T}_{\varphi}^{\mathcal{S}}\left(\theta, \Lambda^{*}\right) & \equiv \sup _{\alpha} \tilde{T}_{\varphi}^{\mathcal{S}}\left(\theta, \alpha, \Lambda^{*}\right),
\end{aligned}
$$

and the same for $\tilde{I}_{\varphi}^{\mathcal{S}}$ and $\tilde{J}_{\varphi}^{\mathcal{S}}$. These quantities have properties similar to those of the CNT entropy $[14,23]$.

\section{Formulation by QMC}

A construction of dynamical entropy is due to the quantum Markov chain [4].

Let $\mathcal{A}$ be a von Neumann algebra acting on a Hilbert space $\mathcal{H}$ and let $\varphi$ be a state on $\mathcal{A}$ and $\mathcal{A}_{0}=M_{d}(d \times d$ matrix algebra $)$. Take the transition expectation $\mathcal{E}_{\gamma}: \mathcal{A}_{0} \otimes \mathcal{A} \rightarrow \mathcal{A}$ of Accardi [1, 2] such that

$$
\mathcal{E}_{\gamma}(\tilde{A})=\sum_{i} \gamma_{i} A_{i i} \gamma_{i}
$$

where $\tilde{A}=\sum_{i, j} e_{i j} \otimes A_{i j} \in \mathcal{A}_{0} \otimes \mathcal{A}$ and $\gamma=\left\{\gamma_{j}\right\}$ is a finite partition of unity $I \in \mathcal{A}$. Quantum Markov chain is defined by $\psi \equiv\left\{\varphi, \mathcal{E}_{\gamma, \theta}\right\} \in \Sigma\left(\stackrel{\infty}{\otimes} \mathcal{A}_{0}\right)$ such that

$$
\psi\left(j_{1}\left(A_{1}\right) \cdots j_{n}\left(A_{n}\right)\right) \equiv \varphi\left(\mathcal{E}_{\gamma, \theta}\left(A_{1} \otimes \mathcal{E}_{\gamma, \theta}\left(A_{2} \otimes \cdots \otimes A_{n-1} \mathcal{E}_{\gamma, \theta}\left(A_{n} \otimes I\right) \cdots\right)\right)\right),
$$


where $\mathcal{E}_{\gamma, \theta}=\theta \circ \mathcal{E}_{\gamma}, \theta \in \operatorname{Aut}(\mathcal{A})$, and $j_{k}$ is an embedding of $\mathcal{A}_{0}$ into $\stackrel{\infty}{\otimes}_{1}^{\infty} \mathcal{A}_{0}$ such that $j_{k}(A)=I \otimes \cdots \otimes I \otimes \underset{k-\text { th }}{A} \otimes I \cdots$

Suppose that for $\varphi$ there exists a unique density operator $\rho$ such that $\varphi(A)=\operatorname{Tr} \rho A$ for any $A \in \mathcal{A}$. Let us define a state $\psi_{n}$ on $\stackrel{n}{\otimes} \mathcal{A}_{0}$ expressed as

$$
\psi_{n}\left(A_{1} \otimes \cdots \otimes A_{n}\right)=\psi\left(j_{1}\left(A_{1}\right) \cdots j_{n}\left(A_{n}\right)\right) .
$$

The density operator $\xi_{n}$ for $\psi_{n}$ is given by

$$
\left.\xi_{n} \equiv \sum_{i_{1}} \cdots \sum_{i_{n}} \operatorname{Tr}_{\mathcal{A}}\left(\theta^{n}\left(\gamma_{i_{n}}\right) \cdots \gamma_{i_{1}} \rho \gamma_{i_{1}} \cdots \theta^{n}\left(\gamma_{i_{n}}\right)\right)\right) e_{i_{1} i_{1}} \otimes \cdots \otimes e_{i_{n} i_{n}} .
$$

Put

$$
\left.P_{i_{n} \cdots i_{1}}=\operatorname{Tr}_{\mathcal{A}}\left(\theta^{n}\left(\gamma_{i_{n}}\right) \cdots \gamma_{i_{1}} \rho \gamma_{i_{1}} \cdots \theta^{n}\left(\gamma_{i_{n}}\right)\right)\right) .
$$

The dynamical entropy through QMC is defined by

$$
\tilde{S}_{\varphi}(\theta ; \gamma) \equiv \limsup _{n \rightarrow \infty} \frac{1}{n}\left(-\operatorname{Tr} \xi_{n} \log \xi_{n}\right)=\limsup _{n \rightarrow \infty} \frac{1}{n}\left(-\sum_{i_{1}, \ldots, i_{n}} P_{i_{n} \ldots i_{1}} \log P_{i_{n} \ldots i_{1}}\right) .
$$

If $P_{i_{n} \cdots i_{1}}$ satisfies the Markov property, then the above equality is written by

$$
\tilde{S}_{\varphi}(\theta ; \gamma)=-\sum_{i_{1}, i_{2}} P\left(i_{2} \mid i_{1}\right) P\left(i_{1}\right) \log P\left(i_{2} \mid i_{1}\right) .
$$

The dynamical entropy through QMC with respect to $\theta$ and a von Neumann subalgebra $\mathcal{B}$ of $\mathcal{A}$ is given by

$$
\tilde{S}_{\varphi}(\theta ; \mathcal{B}) \equiv \sup \left\{\tilde{S}_{\varphi}(\theta ; \gamma): \quad \gamma \subset \mathcal{B}\right\}
$$

\section{Formulation by $\mathbf{A F}$}

Let $\mathcal{A}$ be a $C^{*}$-algebra, $\theta$ be an automorphism on $\mathcal{A}$ and $\varphi$ be a stationary state with respect to $\theta$ and $\mathcal{B}$ be a unital *-subalgebra of $\mathcal{A}$. A set $\gamma=\left\{\gamma_{1}, \gamma_{2}, \ldots, \gamma_{k}\right\}$ of elements of $\mathcal{B}$ is called a finite operational partition of unity of size $k$ if $\gamma$ satisfies the following condition:

$$
\sum_{i=1}^{k} \gamma_{i}^{*} \gamma_{i}=I
$$

The operation $\circ$ is defined by

$$
\gamma \circ \xi \equiv\left\{\gamma_{i} \xi_{j} ; \quad i=1,2, \ldots, k, \quad j=1,2, \ldots, l\right\}
$$

for any partitions $\gamma=\left\{\gamma_{1}, \gamma_{2}, \ldots, \gamma_{k}\right\}$ and $\xi=\left\{\xi_{1}, \xi_{2}, \ldots \xi_{l}\right\}$. For any partition $\gamma$ of size $k$, a $k \times k$ density matrix $\rho[\gamma]=\left(\rho[\gamma]_{i, j}\right)$ is given by

$$
\rho[\gamma]_{i, j}=\varphi\left(\gamma_{j}^{*} \gamma_{i}\right) .
$$


Then the dynamical entropy $\tilde{H}_{\varphi}(\theta, \mathcal{B}, \gamma)$ with respect to the partition $\gamma$ and shift $\theta$ is defined by von Neumann entropy $S(\cdot)$;

$$
\tilde{H}_{\varphi}(\theta, \mathcal{B}, \gamma)=\limsup _{n \rightarrow \infty} \frac{1}{n} S\left(\rho\left[\theta^{n-1}(\gamma) \circ \cdots \circ \theta(\gamma) \circ \gamma\right]\right) .
$$

The dynamical entropy $\tilde{H}_{\varphi}(\theta, \mathcal{B})$ is given by taking the supremum over operational partition of unity in $\mathcal{B}$ as

$$
\tilde{H}_{\varphi}(\theta, \mathcal{B})=\sup \left\{\tilde{H}_{\varphi}(\theta, \mathcal{B}, \gamma) ; \gamma \subset \mathcal{B}\right\}
$$

\section{Relations among the four formulations}

In this section we discuss relations among the above four formulations. The $\mathcal{S}$-mixing entropy in GQS introduced in [20] is

$$
S^{\mathcal{S}}(\varphi)=\inf \left\{H(\mu) ; \quad \mu \in M_{\varphi}(\mathcal{S})\right\}
$$

where $H(\mu)$ is given by

$$
H(\mu)=\sup \left\{-\sum_{A_{k} \in \tilde{A}} \mu\left(A_{k}\right) \log \mu\left(A_{k}\right): \quad \tilde{A} \in P(\mathcal{S})\right\},
$$

and $P(\mathcal{S})$ is the set of all finite partitions of $\mathcal{S}$.

The following theorem $[14,23]$ shows the relation between the formulation by CNT and that by complexity.

THEOREM 5.1. Under the above settings, we have the following relations:

(1) $0 \leq I^{\mathcal{S}}\left(\varphi ; \Lambda^{*}\right) \leq T^{\mathcal{S}}\left(\varphi ; A^{*}\right) \leq J^{\mathcal{S}}\left(\varphi ; \Lambda^{*}\right)$,

(2) $C_{I}^{\bar{\Sigma}}(\varphi)=C_{T}^{\Sigma}(\varphi)=C_{J}^{\Sigma}(\varphi)=S^{\Sigma}(\varphi)=H_{\varphi}(\mathcal{A})$,

(3) $\mathcal{A}=\overline{\mathcal{A}}=B(\mathcal{H})$, for any density operator $\rho$, and

$$
0 \leq I^{\mathcal{S}}\left(\rho ; \Lambda^{*}\right)=T^{\mathcal{S}}\left(\rho ; \Lambda^{*}\right) \leq J^{\mathcal{S}}\left(\rho ; \Lambda^{*}\right) .
$$

Since there exists a model showing that $S^{I(\alpha)}(\varphi) \geq H_{\varphi}\left(\mathcal{A}_{\alpha}\right), S^{\mathcal{S}}(\varphi)$ distinguishes states more sharply than $H_{\varphi}(\mathcal{A})$, where $\mathcal{A}_{\alpha}=\{A \in \mathcal{A} ; \alpha(A)=A\}$.

Furthermore, we have the following results [24].

(1) When $\mathcal{A}_{n}, \mathcal{A}$ are abelian $C^{*}$-algebras and $\alpha_{k}$ is an embedding map, then

$$
\begin{aligned}
T^{\Sigma}\left(\mu ; \alpha^{M}\right) & =S_{\mu}^{\text {classical }}\left(\bigvee_{m=1}^{M} \tilde{A}_{m}\right), \\
I^{\Sigma}\left(\mu ; \alpha^{M}, \bar{\alpha}^{N}\right) & =I_{\mu}^{\text {classical }}\left(\bigvee_{m=1}^{M} \tilde{A}_{m}, \bigvee_{n=1}^{N} \tilde{B}_{n}\right)
\end{aligned}
$$

are satisfied for any finite partitions $\tilde{A}_{m}, \tilde{B}_{n}$ on the probability space $(\Omega=\operatorname{spec}(\mathcal{A})$, $\mathcal{F}, \mu)$. 
(2) When $A$ is the restriction of $\mathcal{A}$ to a subalgebra $\mathcal{M}$ of $\mathcal{A} ; A=\mid \mathcal{M}$,

$$
H_{\varphi}(\mathcal{M})=J^{\Sigma}(\varphi ; \mid \mathcal{M})=J_{\varphi}^{\Sigma}(\mathrm{id} ; \mid \mathcal{M}) .
$$

Moreover, when

$$
\begin{aligned}
\mathcal{N} \subset \mathcal{A}_{0}, \quad \mathcal{A}=\bigotimes^{\mathbb{N}} \mathcal{A}_{0}, \quad \theta \in \operatorname{Aut}(\mathcal{A}) ; \\
\alpha^{N} \equiv\left(\alpha, \theta \circ \alpha, \ldots ; \theta^{N-1} \circ \alpha\right) ; \\
\alpha=\bar{\alpha} ; \quad \mathcal{A}_{0} \rightarrow \mathcal{A} \text { an embedding } \\
\mathcal{N}_{N} \equiv \bigotimes_{1}^{N} \mathcal{N},
\end{aligned}
$$

we have

$$
\tilde{H}_{\varphi}(\theta ; \mathcal{N})=\tilde{J}_{\varphi}^{\Sigma}(\theta ; \mathcal{N})=\limsup _{N \rightarrow \infty} \frac{1}{N} J_{\varphi}^{\Sigma}\left(\alpha^{N} ; \mid \mathcal{N}_{N}\right)
$$

We show the relation between the formulation by complexity and that by QMC. Under the same settings in Section 3, we define a map $\mathcal{E}_{(n, \gamma)}^{*}$ from $\Sigma(\mathcal{H})$, the set of all density operators in $\mathcal{H}$, to $\Sigma\left(\left(\stackrel{n}{\otimes} \mathbb{C}^{d}\right) \otimes \mathcal{H}\right)$ by

$$
\begin{aligned}
\mathcal{E}_{(n, \gamma)}^{*}(\rho)= & \sum_{i_{1}} \cdots \sum_{i_{n-1}} \sum_{i_{n}} e_{i_{1} i_{1}} \otimes \cdots \otimes e_{i_{n-1} i_{n-1}} \otimes e_{i_{n} i_{n}} \otimes \\
& \otimes \theta^{n-1}\left(\gamma_{i_{n}}\right) \theta^{(n-2)}\left(\gamma_{i_{n-1}}\right) \cdots \gamma_{i_{1}} \rho \gamma_{i_{1}} \cdots \theta^{(n-2)}\left(\gamma_{i_{n-1}}\right) \theta^{n-1}\left(\gamma_{i_{n}}\right),
\end{aligned}
$$

for any density operator $\rho \in \Sigma(\mathcal{H})$. Let us take a map $E_{(n)}^{*}$ from $\Sigma\left(\left(\underset{1}{\otimes} \mathbb{C}^{d}\right) \otimes \mathcal{H}\right)$ to $\Sigma\left(\stackrel{n}{\otimes} \mathbb{C}^{d}\right)$ such that

$$
E_{(n)}^{*}(\sigma)=\operatorname{Tr}_{\mathcal{H}} \sigma, \quad \forall \sigma \in \Sigma\left(\left(\bigotimes_{1}^{n} \mathbb{C}^{d}\right) \otimes \mathcal{H}\right)
$$

Then a map $\Gamma_{(n, \gamma)}^{*}$ from $\Sigma(\mathcal{H})$ to $\Sigma\left(\stackrel{n}{\otimes} \mathbb{C}^{d}\right)$ is given by

$$
\Gamma_{(n)}^{*}(\rho) \equiv E_{(n)}^{*}\left(\mathcal{E}_{(n, \gamma)}^{*}(\rho)\right), \forall \rho \in \Sigma(\mathcal{H}),
$$

so that $\Gamma_{(n, \gamma)}^{*}(\rho)=\xi_{n}$ and

$$
\tilde{S}_{\varphi}(\theta ; \gamma)=\limsup _{n \rightarrow \infty} \frac{1}{n} S\left(\Gamma_{(n, \gamma)}^{*}(\rho)\right) .
$$

From Theorem 5.1, we have $C_{I}^{\Sigma}\left(\Gamma_{(n, \gamma)}^{*}(\rho)\right)=S\left(\Gamma_{(n, \gamma)}^{*}(\rho)\right)$. Hence

$$
\tilde{S}_{\varphi}(\theta ; \gamma)=\tilde{C}_{I}^{\Sigma}\left(\Gamma_{(\gamma)}^{*}(\rho)\right)\left(\equiv \underset{n \rightarrow \infty}{\limsup } \frac{1}{n} C_{I}^{\Sigma}\left(\Gamma_{(n, \gamma)}^{*}(\rho)\right)\right) .
$$


Now we briefly show the relation between the formulation by complexity and that by $\mathrm{AF}$.

Under the same settings in Section 4, for any partitions $\gamma=\left\{\gamma_{1}, \cdots, \gamma_{k}\right\}$ of size $k$, the $k \times k$ density matrix $\rho[\gamma]=\left(\rho[\gamma]_{i, j}\right)$ is defined by

$$
\rho[\gamma]_{i, j}=\varphi\left(\gamma_{j}^{*} \gamma_{i}\right)
$$

which is acting on the $k$-dimensional Hilbert space $\mathcal{H}_{k}$. We define a map $\Xi_{(m, \gamma)}^{*}$ from $\Sigma\left(\mathcal{H}_{k}\right)$ to $\Sigma\left(\mathcal{H}_{k^{m}}\right)$ by

$$
\Xi_{(m, \gamma)}^{*}(\rho[\gamma])=\rho\left[\theta^{m-1}(\gamma) \circ \cdots \circ \theta(\gamma) \circ \gamma\right]
$$

for any partitions $\gamma=\left\{\gamma_{1}, \cdots, \gamma_{k}\right\}$ of size $k$ and any density matrices $\rho[\gamma] \in \Sigma\left(\mathcal{H}_{k}\right)$. The dynamical entropy by AF is given by

$$
\tilde{H}_{\varphi}(\theta, \mathcal{B}, \gamma)=\limsup _{m \rightarrow \infty} \frac{1}{m} S\left(\Xi_{(m, \gamma)}^{*}(\rho[\gamma])\right) .
$$

Since $C_{I}^{\Sigma}\left(\Xi_{(m, \gamma)}^{*}(\rho[\gamma])\right)=S\left(\Xi_{(m, \gamma)}^{*}(\rho[\gamma])\right)$, we have

$$
\tilde{H}_{\varphi}(\theta, \mathcal{B}, \gamma)=\tilde{C}_{I}^{\Sigma}\left(\Xi_{(\gamma)}^{*}(\rho[\gamma])\right) \text {. }
$$

In any case, the formulation by the entropic complexities contains other formulations. Moreover it opens other possibilities to classify dynamical systems more fine [5].

\section{Computation of quantum dynamical complexity for a model}

Let $X=\left\{a_{1}, \ldots, a_{M}\right\}$ be an alphabet used to construct the input signals and let $\mathcal{S}=$ $\left\{E_{1}, \ldots, E_{M}\right\}$ be a set of one-dimensional projections on a Hilbert space $\mathcal{H}_{0}$ satisfying (1) $E_{n} \perp E_{m} \quad(n \neq m)$ and (2) $E_{n}$ corresponds to the character $a_{n}$.

By $\Sigma_{0}$ we denote the set of density operators generated by $\mathcal{S}$

$$
\Sigma_{0} \equiv\left\{\sigma=\sum_{n=1}^{M} \lambda_{n} E_{n} ; \quad \sum_{n=1}^{M} \lambda_{n}=1, \quad \lambda \geq 0\right\} .
$$

Suppose that the input quantum state is an element of $\Sigma_{0}$. To send the information effectively, the state is first transmitted through a quantum modulator; the transmitted state is called the quantum modulated state. Let $\gamma_{(\mathcal{M})}^{*}$ be a map from $\Sigma_{0}$ to $\Sigma_{0}^{(\mathcal{M})}$ such that $\gamma_{(\mathcal{M})}$ is a completely positive unital map from $\mathcal{A}$ to $\mathcal{A}$ induced by the modulator $(\mathcal{M})$. For any $E_{n} \in \mathcal{S}$, the modulated state $E_{n}^{(\mathcal{M})}$ is given by

$$
E_{n}^{(\mathcal{M})}=\gamma_{(\mathcal{M})}^{*}\left(E_{n}\right) \text {. }
$$

By $\Sigma_{0}^{(\mathcal{M})}$ we denote the set of modulated states

$$
\Sigma_{0}^{(\mathcal{M})} \equiv\left\{\sigma^{(\mathcal{M})}=\sum_{n=1}^{M} \lambda_{n} E_{n}^{(\mathcal{M})} ; \quad \sum_{n=1}^{M} \lambda_{n}=1, \quad \lambda \geq 0\right\} .
$$

In this paper, we consider the modulated states constructed by the photon number states. 
(1) For any $E_{n} \in \Sigma_{0}$, the modulated state $E_{n}^{(P A M)}$ for PAM (Pulse Amplitude Modulator) is defined by

$$
E_{n}^{(P A M)}=\gamma_{(P A M)}^{*}\left(E_{n}\right)=|n\rangle\langle n|,
$$

where $|n\rangle\langle n|$ is the $n$ photon number state on $\mathcal{H}$.

(2) For any $E_{n} \in \Sigma_{0}$, the modulated state $E_{n}^{(P P M)}$ for PPM (Pulse Position Modulator) is defined by

$$
\begin{aligned}
E_{n}^{(P P M)} & =\gamma_{(P P M)}^{*}\left(E_{n}\right) \\
& =\underbrace{E_{0}^{(P A M)} \otimes \cdots \otimes E_{0}^{(P A M)} \otimes \overbrace{E_{d}^{(P A M)}}^{n-\text { th }} \otimes E_{0}^{(P A M)} \otimes \cdots \otimes E_{0}^{(P A M)}}_{M},
\end{aligned}
$$

where $E_{0}^{(P A M)}$ is the vacuum state and $E_{d}^{(P A M)}=|d\rangle\langle d|$ ( $d$ is fixed).

The transmission efficiency using the MER (mutual entropy - entropy ratio) [29] is calculated for some modulators.

Now we compute the mean dynamical mutual entropy for PAM and PPM expressed by the photon number statc (as above).

Let $B\left(\mathcal{H}_{0}\right)$ (resp. $B\left(\overline{\mathcal{H}}_{0}\right)$ ) be the set of all bounded linear operators on a Hilbert space $\mathcal{H}_{0}$ (resp. $\left.\overline{\mathcal{H}}_{0}\right)$, and let $\mathcal{B}_{0}$ (resp. $\overline{\mathcal{B}}_{0}$ ) be a finite subset in $B\left(\mathcal{H}_{0}\right)$ (resp. $B\left(\overline{\mathcal{H}}_{0}\right)$ ). Let $\mathcal{A}\left(\right.$ resp. $\overline{\mathcal{A}}$ ) be an infinite tensor product space of $B\left(\mathcal{H}_{0}\right)$ (resp. $B\left(\overline{\mathcal{H}}_{0}\right)$ ) denoted by

$$
\mathcal{A} \equiv \bigotimes_{i=-\infty}^{\infty} B\left(\mathcal{H}_{0}\right), \quad \mathcal{A} \equiv \bigotimes_{i=-\infty}^{\infty} B\left(\mathcal{H}_{0}\right)
$$

Moreover, let $\theta$ (resp. $\bar{\theta}$ ) be a shift transformations on $\mathcal{A}($ resp. $\overline{\mathcal{A}})$ defined by

$$
\begin{array}{llll}
\theta\left(\bigotimes_{i=-\infty}^{\infty} A_{i}\right)=\bigotimes_{i^{\prime}=-\infty}^{\infty} A_{i^{\prime}} & \left(i^{\prime}=i-1\right) & \text { for any } & \bigotimes_{i=-\infty}^{\infty} A_{i} \in \mathcal{A}, \\
\bar{\theta}\left(\bigotimes_{j=-\infty}^{\infty} \bar{A}_{j}\right)=\bigotimes_{j^{\prime}=-\infty}^{\infty} \bar{A}_{j^{\prime}} \quad\left(j^{\prime}=j-1\right) & \text { for any } & \bigotimes_{j=-\infty}^{\infty} \bar{A}_{j} \in \overline{\mathcal{A}} .
\end{array}
$$

Let $\alpha$ (resp. $\bar{\alpha}$ ) be the embedding map from $\mathcal{B}_{0}$ into $\mathcal{A}$ (resp. $\overline{\mathcal{B}}_{0}$ to $\overline{\mathcal{A}}$ ) given by

$$
\begin{array}{lll}
\alpha(A)=\cdots I \otimes I \otimes A \otimes I \otimes \cdots \in \mathcal{A}, & \text { for any } & A \in \mathcal{B}_{0}, \\
\bar{\alpha}(\bar{A})=\cdots I \otimes I \otimes \bar{A} \otimes I \otimes \cdots \in \overline{\mathcal{A}}, & \text { for any } & \bar{A} \in \overline{\mathcal{B}}_{0} .
\end{array}
$$

'The set of all density operators on $\mathcal{H}_{0}$ (resp. $\overline{\mathcal{H}}_{0}$ ) we denote by $\Sigma_{0}$ (resp. $\bar{\Sigma}_{0}$ ), and let $\Sigma($ resp. $\bar{\Sigma})$ be the set of all density operators on $\mathcal{A}($ resp. $\bar{\rho}$ on $\overline{\mathcal{A}})$. 
The maps $\alpha_{(\mathcal{M})}^{N}$ and $\bar{\alpha}_{\tilde{\Lambda}(\mathcal{M})}^{N}$ are given by

$$
\begin{aligned}
\alpha_{(\mathcal{M})}^{N} & \equiv\left(\alpha \circ \tilde{\gamma}_{(\mathcal{M})}, \theta \circ \alpha \circ \tilde{\gamma}_{(\mathcal{M})}, \ldots, \theta^{N-1} \circ \alpha \circ \tilde{\gamma}_{(\mathcal{M})}\right) \\
\bar{\alpha}_{\tilde{\Lambda}(\mathcal{M})}^{N} & \equiv\left(\tilde{\gamma}_{(\mathcal{M})} \circ \tilde{\Lambda} \circ \bar{\alpha}, \tilde{\gamma}_{(\mathcal{M})} \circ \tilde{\Lambda} \circ \bar{\theta} \circ \bar{\alpha}, \ldots, \tilde{\gamma}_{(\mathcal{M})} \circ \tilde{\Lambda} \circ \bar{\theta}^{N-1} \circ \bar{\alpha}\right)
\end{aligned}
$$

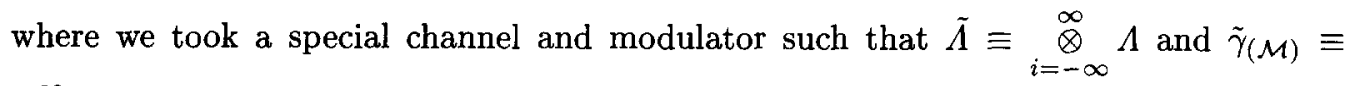
$\stackrel{\infty}{\otimes} \gamma_{(\mathcal{M})}$

(I) PAM. Let us take a stationary initial state $\rho \in \Sigma$

$$
\rho=\sum_{m} \mu_{m} \bigotimes_{i=-\infty}^{\infty} \rho_{m}^{(i)} \quad\left(\sum_{m} \mu_{m}=1, \quad \mu_{m} \neq \mu_{m^{\prime}}, \quad\left(m \neq m^{\prime}\right)\right)
$$

with the unique Schatten decomposition $\rho_{m}^{(i)}=\sum_{n_{i}=1}^{M} \lambda_{n_{i}}^{(m)} E_{n_{i}} \in \Sigma_{0}\left(\sum_{n_{i}=1}^{M} \lambda_{n_{i}}^{(m)}=\right.$ $\left.1, \lambda_{n_{i}}^{(m)} \neq \lambda_{k_{i}}^{(m)},\left(n_{i} \neq k_{i}\right)\right)$ of $\rho_{m}^{(i)}$. Then we have

$$
\begin{aligned}
\Phi_{E}\left(\alpha_{(P A M)}^{N}\right) & =\sum_{n_{0}=1}^{M} \cdots \sum_{n_{N-1}=1}^{M}\left(\sum_{m} \mu_{m} \prod_{k=0}^{N-1} \lambda_{n_{k}}^{(m)}\right)\left(\bigotimes_{i=0}^{N-1} E_{n_{i}}^{(P A M)}\right) \\
\Phi_{E}\left(\bar{\alpha}_{\tilde{\Lambda}(P A M)}^{N}\right) & =\sum_{n_{0}=1}^{M} \cdots \sum_{n_{N-1}=1}^{M}\left(\sum_{m} \mu_{m} \prod_{k=0}^{N-1} \lambda_{n_{k}}^{(m)}\right)\left(\bigotimes_{i-0}^{N-1} \Lambda^{*} E_{n_{i}}^{(P A M)}\right) .
\end{aligned}
$$

When $\Lambda^{*}$ is an attenuation channel, we obtain [17]

$$
\Lambda^{*} E_{n_{i}}^{(P A M)}=\sum_{j_{i}=0}^{n_{i}}\left|C_{j_{i}}^{n_{i}}\right|^{2} F_{j_{i}}^{(P A M)}
$$

where $F_{j_{i}}^{(P A M)}=\left|j_{i}\right\rangle\left\langle j_{i}\right|$ is the $j_{i}$-photon number state in the output space $\bar{\Sigma}_{0}$ and

$$
\left|C_{j_{i}}^{n_{i}}\right|^{2}=\frac{n_{i} !}{j_{i} !\left(n_{i}-j_{i}\right) !} \eta^{j_{i}}(1-\eta)^{\left(n_{i}-j_{i}\right)}
$$

where $\eta$ is the transmission rate of the channel $[17,19]$. The compound states related to the channel $\tilde{\Lambda}^{*}$ become

$$
\begin{aligned}
\Phi_{E}\left(\alpha_{(P A M)}^{N} \cup \bar{\alpha}_{\tilde{\Lambda}(P A M)}^{N}\right) & =\sum_{n_{0}=1}^{M} \ldots \sum_{n_{N-1}=1}^{M}\left(\sum_{m} \mu_{m} \prod_{k=0}^{N-1} \lambda_{n_{k}}^{(m)}\right) \sum_{j_{0}=0}^{n_{0}} \ldots \sum_{j_{N-1}=0}^{n_{N-1}}\left(\prod_{k^{\prime}=0}^{N-1}\left|C_{j_{k^{\prime}}}^{n_{k^{\prime}}}\right|^{2}\right) \times \\
& \times\left(\bigotimes_{i=0}^{N-1} E_{n_{i}}^{(P A M)}\right)\left(\bigotimes_{i^{\prime}=0}^{N-1} F_{j_{i^{\prime}}}^{(P A M)}\right)
\end{aligned}
$$




$$
\begin{aligned}
& \Phi_{E}\left(\alpha_{(P A M)}^{N}\right) \otimes \Phi_{E}\left(\bar{\alpha}_{\tilde{\Lambda}(P A M)}^{N}\right) \\
&=\sum_{n_{0}=1}^{M} \ldots \sum_{n_{N-1}=1}^{M}\left(\sum_{m} \mu_{m} \prod_{k=0}^{N-1} \lambda_{n_{k}}^{(m)}\right) \sum_{n^{\prime}=1}^{M} \ldots \sum_{n^{\prime}{ }_{N-1}=1}^{M}\left(\sum_{m^{\prime}} \prod_{k^{\prime}=0}^{N-1} \lambda_{n^{\prime} k^{\prime}}^{\left(m^{\prime}\right)}\right) \times \\
& \times \sum_{j^{\prime}=0}^{n_{0}=0} \ldots \sum_{j^{\prime}{ }_{N-1}=0}^{n^{\prime}{ }^{N-1}}\left(\prod_{k^{\prime}=0}^{N-1}\left|C_{j^{\prime} k^{\prime \prime}}^{n^{\prime} k^{\prime \prime}}\right|^{2}\right)\left(\bigotimes_{i=0}^{N-1} E_{n_{i}}^{(P A M)}\right)\left(\bigotimes_{i^{\prime}=0}^{N-1} F_{j^{\prime} i^{\prime}}^{(P A M)}\right)
\end{aligned}
$$

For an initial state $\rho$ in $(6.1)$, we have

$$
\begin{aligned}
& \mathrm{I}_{E}\left(\rho ; \alpha_{(P A M)}^{N}, \bar{\alpha}_{\tilde{\Lambda}(P A M)}^{N}\right) \\
& =\sum_{j_{0}=0}^{M} \ldots \sum_{j_{N}-1=0}^{M} \sum_{n_{0}=J_{0}}^{M} \ldots \sum_{n_{N}-1=J_{N-1}}^{M}\left(\sum_{m} \mu_{m} \prod_{k=0}^{N-1} \lambda_{n_{k}}^{(m)}\right)\left(\prod_{k^{\prime}=0}^{N-1}\left|C_{j_{k^{\prime}}}^{n_{k^{\prime}}}\right|^{2}\right) \times \\
& \times \log \frac{\prod_{k=0}^{N-1}\left|C_{j_{k}}^{n_{k}}\right|^{2}}{\sum_{n^{\prime}{ }_{0}=J_{0}}^{M} \cdots \sum_{n^{\prime} N-1=J_{N-1}}^{M}\left(\sum_{m^{\prime}} \mu_{m^{\prime}} \prod_{k^{\prime}=0}^{N-1} \lambda_{n^{\prime}{ }_{k^{\prime}}}^{\left(m^{\prime}\right)}\right)\left(\prod_{k^{\prime \prime}=0}^{N-1} \mid C_{j_{k^{\prime}}{ }^{\prime \prime}}^{\left.n^{\prime}\right|^{2}}\right)} .
\end{aligned}
$$

Having the above equality, we obtain the following theorem.

THEOREM 6.1. (1) For an initial state $\rho$ in (6.1), we have the lower bound of $\tilde{C}_{\rho}(\theta$, $\left.\alpha_{(P A M)}\right)$ such that

$$
\tilde{C}_{\rho}\left(\theta, \alpha_{(P A M)}\right) \geq \sum_{m} \mu_{m} S\left(\rho_{m}^{(0)}\right) .
$$

(2) Let $\mu_{0}=1, \mu_{k}=0(\forall k \geq 1)$ and $\lambda_{n}^{(0)}=\lambda_{n}$ in (6.1). When $\mathcal{A}=\overline{\mathcal{A}}, \theta=\theta$ and $\alpha=\bar{\alpha}$, we obtain the following equalities:

$$
\begin{aligned}
\tilde{C}_{\rho}\left(\theta, \alpha_{(P A M)}\right) & =-\sum_{n=1}^{M} \lambda_{n} \log \lambda_{n} \\
\tilde{I}_{\rho}^{\Sigma}\left(\theta, \alpha_{(P A M)}, \Lambda^{*}\right) & =\sum_{j=0}^{M} \sum_{n=J}^{M} \lambda_{n}\left|C_{j}^{n}\right|^{2} \log \frac{\left|C_{j}^{n}\right|^{2}}{\sum_{n^{\prime}=J}^{M} \lambda_{n^{\prime}}\left|C_{j}^{n^{\prime}}\right|^{2}} .
\end{aligned}
$$

(II) PPM. For an initial state $\rho$ in (6.1), we obtain the following compound states:

$$
\begin{gathered}
\Phi_{E}\left(\alpha_{(P P M)}^{N}\right)=\sum_{n_{0}=1}^{M} \ldots \sum_{n_{N-1}=1}^{M}\left(\sum_{m} \mu_{m} \prod_{k=0}^{N-1} \lambda_{n_{k}}^{(m)}\right)\left(\bigotimes_{i=0}^{N-1}\left(\bigotimes_{j=0}^{N-1} E_{d \delta_{j, n_{i}}}^{(P A M)}\right)\right) \\
\Phi_{E}\left(\bar{\alpha}_{\tilde{\Lambda}(P P M)}^{N}\right)=\sum_{n_{0}=1}^{M} \ldots \sum_{n_{N-1}=1}^{M}\left(\sum_{m} \mu_{m} \prod_{k=0}^{N-1} \lambda_{n_{k}}^{(m)}\right) \times \\
\times \sum_{\ell_{0}=0}^{d \delta_{1, n_{0}}} \ldots \sum_{\ell_{N-1}=0}^{d \delta_{M, n_{N-1}}}\left|C_{\ell_{0}}^{d \delta_{1, n_{0}}}\right|^{2} \ldots\left|C_{\ell_{N-1}}^{d \delta_{M, n_{N}-1}}\right|^{2}\left(\bigotimes_{i=0}^{N-1}\left(\bigotimes_{j=1}^{M} F_{\ell_{i M+j}}^{(P A M)}\right)\right) .
\end{gathered}
$$




$$
\begin{aligned}
\Phi_{E}\left(\alpha_{(P P M)}^{N} \cup \bar{\alpha}_{\tilde{\Lambda}(P P M)}^{N}\right) & \\
= & \sum_{n_{0}=1}^{M} \cdots \sum_{n_{N-1}=1}^{M}\left(\sum_{m} \mu_{m} \prod_{k=0}^{N-1} \lambda_{n_{k}}^{(m)}\right) \sum_{\ell_{1}=0}^{d \delta_{1, n_{0}}} \cdots \sum_{\ell_{N-1}=0}^{d \delta_{M, n_{N-1}}}\left|C_{\ell_{1}}^{d \delta_{1, n_{0}}}\right|^{2} \cdots \mid C_{\ell_{N-1}}^{\left.d \delta_{M, n_{N-1}-1}\right|^{2} \times} \\
& \times\left(\bigotimes_{i=0}^{N-1}\left(\bigotimes_{j=1}^{M} E_{d \delta_{j, n_{i}}}^{(P A M)}\right)\right)\left(\bigotimes_{i^{\prime}=0}^{N-1}\left(\bigotimes_{j^{\prime}=0}^{M} F_{\ell_{i^{\prime}} M+j^{\prime}}^{(P A M)}\right)\right)
\end{aligned}
$$

$\Phi_{E}\left(\alpha_{(P P M)}^{N}\right) \otimes \Phi_{E}\left(\bar{\alpha}_{\tilde{\Lambda}(P P M)}^{N}\right)$

$$
\begin{aligned}
& =\sum_{n_{0}=1}^{M} \cdots \sum_{n_{N-1}=1}^{M} \sum_{n^{\prime}=1}^{M} \cdots \sum_{n^{\prime} N}^{M}\left(\sum_{m} \mu_{m} \prod_{k=0}^{N-1} \lambda_{n_{k}}^{(m)}\right) \times \\
& \times \sum_{\ell_{1}=0}^{d \delta_{1, n_{0}}} \ldots \sum_{\ell_{N-1}=0}^{d \delta_{M_{1}, n_{N}-1}}\left|C_{\ell,}^{d \delta_{1, n_{0}}}\right|^{2} \ldots\left|C_{\ell_{N-1}}^{d \delta_{M, n_{N-1}}}\right|^{2} \sum_{n^{\prime}=1}^{M} \ldots \sum_{n^{\prime}{ }_{N-1}=1}^{M}\left(\sum_{m^{\prime}} \mu_{m^{\prime}} \prod_{k^{\prime}=0}^{N-1} \lambda_{n^{\prime}{ }_{k^{\prime}}}\right) \times \\
& \times\left(\bigotimes_{i=0}^{N-1}\left(\bigotimes_{j=1}^{M} E_{d \delta_{j, n_{i}}}^{(P A M)}\right)\right)\left(\bigotimes_{i^{\prime}=0}^{N-1}\left(\bigotimes_{j^{\prime}=1}^{M} F_{\ell_{i^{\prime} M+j^{\prime}}}^{(P A M)}\right)\right) .
\end{aligned}
$$

For an initial state $\rho$ in (6.1), we have

$$
\begin{aligned}
\mathbf{I}_{E}\left(p ; \alpha_{(P P M)}^{N}, \bar{\alpha}_{\tilde{\Lambda}(P P M)}^{N}\right) & \\
= & -\sum_{n_{0}=1}^{M} \cdots \sum_{n_{N-1}=1}^{M}\left(\sum_{m} \mu_{m} \prod_{k=0}^{N-1} \lambda_{n_{k}}^{(m)}\right) \sum_{p=1}^{N} \sum_{\left\{\gamma_{1}, \cdots, q_{p}\right\} \subset\{1,2, \cdots, N\}} \times \\
& \times \sum_{\ell_{1}=1}^{d} \cdots \sum_{\ell_{p}=1}^{d}\left|C_{\ell_{1}}^{d}\right|^{2} \cdots\left|C_{\ell_{p}}^{d}\right|^{2}(1-\eta)^{N-p} \eta^{p} \log \left(\sum_{m^{\prime}} \mu_{m^{\prime}} \prod_{k^{\prime}=0}^{p} \lambda_{n_{k_{k^{\prime}}}}^{\left(m^{\prime}\right)}\right) .
\end{aligned}
$$

THEOREM 6.2. (1) For an initial state $\rho$ in $(6.1)$, we have the lower bound of $\tilde{C}_{\rho}(\theta$, $\left.\alpha_{(P P M)}\right)$ such that

$$
\bar{C}_{\rho}\left(\theta, \alpha_{(P P M)}\right) \geq \sum_{m} \mu_{m} S\left(\rho_{m}^{(0)}\right) .
$$

(2) Let $\mu_{0}=1, \mu_{k}=0(\forall k \geq 1)$ and $\lambda_{n}^{(0)}=\lambda_{n}$ in (6.1). When $\mathcal{A}=\overline{\mathcal{A}}, \theta=\bar{\theta}$ and $\alpha=\bar{\alpha}$, we obtain the following equalities:

$$
\begin{aligned}
\tilde{C}_{\rho}\left(\theta, \alpha_{(P P M)}\right) & =-\sum_{n=1}^{M} \lambda_{n} \log \lambda_{n}, \\
\tilde{I}_{\rho}^{\Sigma}\left(\theta, \alpha_{(P P M)}, \Lambda^{*}\right) & =\left(1-(1-\eta)^{d}\right) \tilde{C}_{\rho}\left(\theta, \alpha_{(P P M)}\right) .
\end{aligned}
$$

(3) If $\mathcal{A}=\overline{\mathcal{A}}, \theta=\bar{\theta}, \alpha=\bar{\alpha}$ and $d \geq N$, then

$$
\tilde{I}_{\rho}^{\Sigma}\left(\theta, \alpha_{(P P M)}, \Lambda^{*}\right) \geq \tilde{I}_{\rho}^{\Sigma}\left(\theta, \alpha_{(P A M)}, A^{*}\right) .
$$




\section{REFERENCES}

[1] L. Accardi: Noncommutative Markov chains, Internatinal School of Mathematical Physics, Camerino (1974), 268.

[2] L. Accardi, A. Frigerio and J. Lewis: Quantum stochastic processes, Publications of the Research institute for Mathematical Sciences Kyoto University, 18 (1982), 97.

[3] L. Accardi and M. Ohya: Compound channels, transition expectations and liftings, to appear in J. Multivariate Analysis.

[4] L. Accardi, M. Ohya and N. Watanabe: Dynamical entropy through quantum Markov chain, to appear in Open System and Information Dynamics.

[5] R. Alicki and M. Fannes: Lett. Math. Phys. 32 (1994), 75.

[6] H. Araki: Relative entropy for states of von Neumann algebras, Publications of the Research institute for Mathematical Sciences Kyoto University 11 (1976), 809.

[7] F. Benatti: Deterministic Chaos in Infinite Quantum Systems, Springer, Berlin 1993.

[8] L. Bilingsley: Ergodic Theory and Information, Wiley, New York 1965.

[9] A. Connes, H. Narnhoffer and W. Thirring: Commun. Math. Phys. 112 (1987), 691.

[10] A. Connes and E. Størmer: Acta Math. 134 (1975), 289.

[11] G. G. Emch: Z. Wahrscheinlichkeitstheorie verw. Gebiete 29 (1974), 241.

[12] L. Feinstein: Foundations of Information Theory, MacGrow-Hill, 1965.

[13] A. N. Kolmogorov: Dokl. Akad. Nauk SSSR 119 (1958), 861.

[14] N. Muraki and M. Ohya: Lett. Math. Phys. 36 (1996), 327.

[15] J. von Neumann: Die Mathematischen Grundlagen der Quantenmechanik, Springer, Berlin 1932.

[16] M. Ohya: J. Math. Anal. Appl. 84 (1981), 318.

[17] M. Ohya: IEEE Trans. Information Theory 29 (1983), 770.

[18] M. Ohya: L. Nuovo Cimento 38 (1983), 402.

[19] M. Ohya: J. Math. Anal. Appl. 100 (1984), 222.

[20] M. Ohya: Rep. Math. Phys. 27 (1989), 19.

[21] M. Ohya: Information dynamics and its application to optical communication processes, Springer Lecture Notes in Physics, 378, p. 81, Springer, Berlin 1991.

[22] M. Ohya and D. Petz: Quantum Entropy and its Use, Springer, Berlin 1993.

[23] M. Ohya: Quantum Communications and Measurement 2 (1995), 309.

[24] M. Ohya: Foundation of entropy, complexity and fractal in quantum systems, to appear in International Congress of Probability Towards 2000, 1996.

[25] M. Ohya and N. Watanabe: Note on irreversible dynamics and quantum information, to appear in the Alberto Frigerio Conference Proceedings.

[26] J. G. Sinai: Dokl. Akad. Nauk SSSR 124 (1959), 768.

[27] A. Uhlmann: Commun. Math. Phys. 54 (1977), 21.

[28] H. Umegaki: Kodai Math. Sem. Rep. 14 (1962), 59.

[29] N. Watanabe: Quantum Probability and Related Topics 6 (1991), 489. 